In Crescendo, 2019; 10(1): 35-42

Fecha de recepción: 12 de enero del 2019

Fecha de aceptación: 25 de marzo del 2019

\title{
LA MATEMÁTICA RECREATIVA COMO ESTRATEGIA DE APRENDIZAJE
}

\author{
RECREATIONAL MATH AS A \\ LEARNING STRATEGY \\ Victoria Castro ${ }^{1}$ Isabel Menacho-Vargas ${ }^{2}$, \\ Luis Fernando Velarde-Vela ${ }^{3}$
}

\section{RESUMEN}

La investigación tuvo como objetivo general determinar si la aplicación de actividades lúdicas como estrategia mejora el aprendizaje de las matemáticas en los estudiantes de primaria de la Institución Educativa Naciones Unidas N`7062 en el año 2017. Esta investigación fue preexperimental, de enfoque cuantitativo. Se realizó con una muestra de 26 estudiantes a los cuales se les aplicó un pretest y postest. Se utilizó la estadística descriptiva para la interpretación de las variables. Asimismo, se utilizó la estadística inferencial con el estadístico de Wilcoxon para la prueba de hipótesis, en la cual se pudo apreciar el valor de $\mathrm{P}=, 000<0,05$; es decir que existe una mejora significativa en el aprendizaje de las matemáticas después de la aplicación de las actividades lúdicas. Se concluyó en que la aplicación de actividades lúdicas como estrategia mejora significativamente el aprendizaje de las matemáticas en los estudiantes de primaria de la Institución Educativa Naciones Unidas N7062 en el año 2017.

PAlabras ClaVE: Actividades lúdicas, Estrategia, Aprendizaje de las matemáticas.

1 Bachiller en Matemática - Física de la Universidad Nacional Federico Villarreal. Magíster en Docencia y gestión educativa de la Universidad César Vallejo.

2 Licenciada en Psicología y en Educación, UNMSM, UIGV. Doctora en Administración de la Educación de la Universidad César Vallejo.

3 Licenciado en Matemática - Física de la Universidad Federico Villarreal. Doctor en Educación de la Universidad César Vallejo. 


\begin{abstract}
The general objective of the research was to determine whether the application of recreational activities as a strategy improves the learning of mathematics in elementary students of the United Nations Educational Institution No. 7062 in the year 2017. This research was preexperimental, with a quantitative approach. It was carried out with a sample of 26 students to whom a pre-test and post-test were applied. Descriptive statistics was used for the interpretation of the variables. Likewise, inferential statistics were used with the Wilcoxon statistic for the hypothesis test, in which the value of $\mathrm{P}=, 000<0.05$; that is, there is a significant improvement in the learning of mathematics after the application of play activities. It was concluded that the application of recreational activities as a strategy significantly improves the learning of mathematics in elementary students of the Educational Institution United Nations No. 7062 in the year 2017.
\end{abstract}

KEY WORDS: Play activities, Strategy, Learning mathematics.

\title{
INTRODUCCIÓN
}

La matemática es una de las asignaturas que se enseña en todos los países del mundo y también en los diversos niveles que hay, lamentablemente no en todos los países, se enseña de la manera más adecuada y tampoco le dan la importancia que la requiere. Porque al hablar de Matemática estamos haciendo referencia a un área muy compleja, pero a su vez muy buena, que permite desarrollar como menciona Rodríguez (2006) el pensamiento lógico en los estudiantes, y también permite solucionar problemas de la vida cotidiana.

Según la Unesco (2015), menciona que, nuestro país obtuvo una mejora en sus resultados, que fue uno de los países que más subió el promedio en relación a otros, cabe resaltar, que, por más que se haya mejorado, igual seguimos ocupando los últimos lugares en las evaluaciones matemáticas.

Según el Minedu (2012), el aprendizaje de la matemática está vinculado con el acrecentamiento de capacidades, conocimientos y actitudes matemáticas además del desarrollo del pensamiento matemático. Por eso, Muñiz, Alonso y Rodríguez (2014) afirman que centrar las clases en actividades lúdicas contribuyen a desarrollar en el alumnado mucho más dichas capacidades matemáticas que marca el currículo.

Ferrero (1991) y Sarlé (2006) desde el punto de vista de desarrollo intelectual, nos dice que el juego es una excelente actividad para ejercitar las capacidades mentales que al igual que las físicas, se mejoran con el ejercicio, con la prácti- 
ca, estimula la imaginación, enseña a pensar con espíritu crítico, favorece la creatividad. Todo esto mencionado es un trabajo muy arduo para los docentes y sobre todo para los niños, porque depende de varios factores el poder hacer esto realidad. En el caso de los docentes, dice Colmenares (2009) que tienen que buscar diversas estrategias activas y novedosas que permita captar la atención del estudiante. Al respecto también nos menciona Medina (2016) que para el aprendizaje de la matemática se deben plantear actividades prácticas y recreativas, ya que esta no es una materia de mucho agrado para la mayoría, por ende, dichas estrategias tienen que ser motivadoras y que incentiven el interés por aprenderla.

Para el estudiante muchas veces la matemática resulta ser una materia aburrida, difícil y poco interesante, puede ser porque los docentes que han tenido la han dictado de la forma tradicional, en donde el estudiante es un receptor pasivo y el docente solo llena la pizarra de ejercicios y deja que ellos solos resuelvan. Por eso también es necesario como lo afirma Ciro y Torres (2016) que el docente debe utilizar la literatura para aportar belleza y encanto a la enseñanza de la matemática y puede ser también un instrumento para recrear este mundo. Por tal motivo esta investigación se basa en los estudios de Gardner (1975) quién se dedicó durante muchos años al aporte de las matemáticas recreativas y afirma que, para hacer las matemáticas interesantes para los alumnos, es acercarse a ellas en son de juego y que el mejor método para mantener despierto a un estudiante es proponerle un juego matemático y de Piaget (1976) quien menciona que las operaciones lógico-matemáticas, antes de ser una actitud puramente intelectual, requiere en el preescolar la construcción de estructuras internas y del manejo de ciertas nociones que son, ante todo, producto de la acción y relación del niño con objetos y sujetos. El adulto que acompaña al niño en su proceso de aprendizaje debe planificar didáctica de procesos que le permitan interaccionar con objetos reales, que sean su realidad: personas, juguetes, ropa, animales, plantas, etc. Según Catillo (2008), menciona que Piaget considera dos poderosos motores que hacen que el ser humano mantenga ese desarrollo continuo de sus estructuras cognitivas: la adaptación y el acomodamiento al conjugar estos elementos, se puede conocer la importancia de vincular un marco teórico con la práctica pedagógica que ha de ejercer un docente al enseñar los contenidos matemáticos en el aula.

Debido a lo escrito líneas arriba es que nos planteamos la siguiente pregunta: ¿De qué manera la aplicación de actividades lúdicas como estrategia mejora el aprendizaje de las matemáticas en los estudiantes de primaria de la Institución Educativa Naciones Unidas N7062 en el año 2017? 
Son muchos los investigadores que afirman en sus investigaciones, que la aplicación de diversas actividades didácticas utilizando material concreto, mejora significativamente el aprendizaje en el área de Matemática de los estudiantes como Lezama y Tamayo (2012); Alcocer F. y Alcocer M. (2016) y Zapata, Colorado y Gutiérrez (2016). Debido a esto es que la presente investigación empieza a tener claro su objetivo que es determinar si la aplicación de actividades lúdicas como estrategia mejora el aprendizaje de las matemáticas en los estudiantes de primaria de la Institución Educativa Naciones Unidas N7062 en el año 2017. Y la hipótesis: La aplicación de actividades lúdicas como estrategia mejora el aprendizaje de las matemáticas en los estudiantes de primaria de la Institución Educativa Naciones Unidas N7062 en el año 2017.

\section{METODOLOGÍA}

La presente investigación fue de tipo aplicada, de diseño experimental. Según Arias (1999) la investigación experimental es todo un proceso en el cual un grupo de individuos se someten a determinados estímulos, para posteriormente observar los efectos que se pueden producir en ellos. En esta investigación por ser preexperimental, se trabajó con un solo grupo de 26 estudiantes.

Es de enfoque cuantitativo porque según Hernández, Fernández y Baptista (2014) cada etapa precede a la siguiente y no podemos eludir pasos. Es de orden riguroso, presenta objetivos y pregunta de investigación, se revisa la literatura y se construye un marco teórico de acorde a nuestras variables.

La población estuvo conformada por 150 estudiantes del sexto grado de educación primaria, con una muestra no probabilística de 26 estudiantes, tomados de la Institución Educativa Naciones Unidas N7062 del distrito de San Juan de Miraflores.

Para la recolección de datos se aplicó la técnica de la Evaluación, y utilizando como instrumentos dos pruebas de Matemática. Dichos instrumentos han sido validados por expertos (Ver Cuadro 1).

Asimismo, se utilizó la estadística no paramétrica de Wilcoxon para la contrastación de hipótesis, para poder comparar mis dos resultados y determinar si existen diferencias entre ellos. 
Cuadro 1

VALIDACIÓN DEL INSTRUMENTO

\begin{tabular}{lccc}
\hline Criterios & Exp. 1 & Exp. 2 & Exp.3 \\
\hline Pertinencia & Aplicable & Aplicable & Aplicable \\
Relevancia & Aplicable & Aplicable & Aplicable \\
Claridad & Aplicable & Aplicable & Aplicable \\
\hline
\end{tabular}

\section{RESULTADOS}

Se presentan los resultados en tablas:

En la tabla 1 en el pretest se observa que el 100\% de estudiantes se encuentran en el nivel Inicio, mientras que los demás niveles se encuentran con $0 \%$ de estudiantes.

En el postest observamos que en el nivel inicio se encuentran 11,5\%; en el nivel proceso un $15,4 \%$; en el nivel logro esperado un $61,5 \%$; y en el nivel logro destacado también un 11,5\%.

Podemos decir que después de la aplicación de las actividades lúdicas los resultados fueron óptimos en la primera prueba de Matemática.

Tabla 1

RESULTADO DE LA PRIMERA PRUEBA PRETEST Y POSTEST DE MATEMÁTICA

\begin{tabular}{lrrrrrr}
\hline & \multicolumn{2}{c}{ Pretest } & & \multicolumn{2}{c}{ Postest } \\
\cline { 2 - 3 } \cline { 6 - 7 } Niveles & Frecuencia & Porcentaje & & Frecuencia & Porcentaje \\
\hline Inicio & 26 & 100,0 & & 3 & 11,5 \\
Proceso & 0 & 0 & & 4 & 15,4 \\
Logro esperado & 0 & 0 & 16 & 61,5 \\
Logro destacado & 0 & 0 & 3 & 11,5 \\
\hline Total & 26 & 100,0 & 26 & 100,0 \\
\hline
\end{tabular}


Tabla 2

RESULTADO DE LA SEGUNDA PRUEBA PRETEST Y POSTEST DE MATEMÁTICA

\begin{tabular}{lrrrrr}
\hline & \multicolumn{2}{c}{ Pretest } & & \multicolumn{2}{c}{ Postest } \\
\cline { 2 - 3 } \cline { 6 - 6 } Niveles & Frecuencia & Porcentaje & & Frecuencia & Porcentaje \\
\hline Inicio & 25 & 96,2 & & 3 & 11,5 \\
Proceso & 1 & 3,8 & & 9 & 34,6 \\
Logro esperado & 0 & 0 & & 12 & 46,2 \\
Logro destacado & 0 & 0 & & 2 & 7,7 \\
\hline Total & 26 & 100,0 & & 26 & 100,0 \\
\hline
\end{tabular}

En la tabla 2 en el pretest se observa que el 96,2\% de estudiantes se encuentran en el nivel Inicio y un 3,8\% en el nivel proceso, mientras que los demás niveles se encuentran con $0 \%$ de estudiantes.

En el postest observamos que en el nivel inicio se encuentran 11,5\%; en el nivel proceso un $34,6 \%$; en el nivel logro esperado un $46,2 \%$; y en el nivel logro destacado también un 7,7\%.

Podemos decir que después de la aplicación de las actividades lúdicas los resultados fueron óptimos en la primera segunda prueba de Matemática.

Para contrastar las hipótesis se realizó: el estadístico de Wilcoxon para datos no paramétricos.

Tabla 3

PRUEBA DE WILCOXON PARA PROBAR LA HIPÓTESIS GENERAL SEGÚN RANGOS ESTADÍSTICOS DE CONTRASTE

\begin{tabular}{|c|c|c|}
\hline Estadísticos de contraste $^{a}$ & $\begin{array}{c}\text { Primera prueba } \\
\text { post - pre }\end{array}$ & $\begin{array}{c}\text { Segunda prueba } \\
\text { post - pre }\end{array}$ \\
\hline PZ & $-4,377 b$ & $-4,211 b$ \\
\hline Sig. asintót. (bilateral) & ,000 &, 000 \\
\hline
\end{tabular}

a. Prueba de los rangos con signo de Wilcoxon.

b. Basado en los rangos negativos. 
En los resultados mostrados en la tabla 3 tanto en el pretest como en el postest de ambas pruebas, el valor de p (Sig. asintót. (bilateral)) es ,000 menor a $\alpha=0,05$; por lo tanto, se rechaza la hipótesis nula y se acepta la $\mathrm{Hi}$, esto quiere decir que después de la aplicación de las actividades lúdicas se mejora significativamente el aprendizaje de las matemáticas en los estudiantes de primaria de la institución educativa Naciones Unidas N7062 en el año 2017.

\section{DISCUSIÓN}

Los resultados de la primera prueba de Matemática antes y después de la aplicación de las actividades lúdicas, muestran en cuanto al pretest, el 100\% de los estudiantes salió desaprobado, quiere decir que se encuentra en el nivel inicio, pero en el postest observamos una notable mejora, en el nivel inicio se encuentran 11,5\%; en el nivel proceso un 15,4\%; en el nivel logro esperado un $61,5 \%$; y en el nivel logro destacado también un 11,5\%.

Los resultados de la segunda prueba de Matemática antes y después de la aplicación de las actividades lúdicas. Observamos en cuanto al pretest el 96.2\% de los estudiantes se encuentra en el nivel inicio y un $3.8 \%$ se encuentra en proceso, pero en el postest observamos una notable mejora, en el nivel inicio se encuentran 11,5\%; en el nivel proceso un 34,6\%; en el nivel logro esperado un 46,2\%; y en el nivel logro destacado también un 7,7\%.

Estos resultados son semejantes a los encontrados en Lezama y Tamayo (2012), en su investigación sobre La aplicación de los juegos didácticos basados en el enfoque significativo mejora el logro de aprendizaje en el área de matemática, donde se encontró en el pretest que un 92\% de los estudiantes se encuentra en el nivel inicio, mientras que en el postets el 100\% obtuvo el más alto nivel de logro previsto.

Asimismo, Muñiz, Alonso y Rodríguez (2014) afirman a partir de sus resultados, un aumento de la motivación y del interés hacia las matemáticas con la implantación de la nueva metodología. Los alumnos afirman que aprender matemáticas jugando les resulta interesante y divertido, y que ha facilitado la comprensión de los conceptos.

\section{CONCLUSIÓN}

Se acepta la hipótesis de investigación, la aplicación de las diversas actividades lúdicas durante el desarrollo de las clases de matemáticas, mejoró significativamente en el aprendizaje de dicha materia en los estudiantes de primaria de la institución educativa Naciones Unidas Nº7062 en el año 2017. 


\section{REFERENCIAS BIBLIOGRÁFICAS}

Alcocer T., Geovanny M., Alcocer T., y Arnaldo F. (2016). Las estrategias recreativas para desarrollar las macro destrezas en el área de matemática, en los niños de quinto año de educación básica, de la unidad educativa “21 de abril”, ubicada en la parroquia flores, cantón Riobamba, provincia de Chimborazo, periodo 2014-2015 (Bachelor's thesis, Riobamba, UNACH 2016).

Arias F. (1999). El proyecto de investigación. Fidias G. Arias Odón.

Boletín del laboratorio Latinoamericano de evaluación de la calidad d la educación Llece 2015 $\mathrm{N}^{\circ} 17$ junio noviembre 2015.

Castillo S. (2008). Propuesta pedagógica basada en el constructivismo para el uso óptimo de las TIC en la enseñanza y el aprendizaje de la matemática. Revista latinoamericana de investigación en matemática educativa, 11(2), 171-194.

Ciro R. y Torres M. (2016). Literatura, matemática y razonabilidad: Una relación triádica en la didáctica de la matemática. Uni-pluri/versidad, 16(1), 34-50. Retrieved from https://search.proquest.com/docview/1872227697? accountid=37408

Colmenares X. (2009). La lúdica en el aprendizaje de las matemáticas. Zona Próxima, (10) Retrieved from https://search.proquest.com/docview/1435680916? accountid=37408

Ferrero L. (1991). El juego y la matemática. Editorial La Muralla.

Gadner M., Carnaval Matemático. Alianza Editorial. Madrid 1975.pg. 8.

Hernández, S., Fernández, C. y Baptista, L. (2014). Metodología de la investigación. México: Mc Graw Hill.

Lezama J. y Tamayo C. 2012. La aplicación de los juegos didácticos basados en el enfoque significativo mejora el logro de aprendizaje en el área de Matemática. Revista In Crescendo. Perú. 3(1), 23-29.

Medina M. (2016). El maravilloso mundo de los números. Educatio Siglo XXI, 34(2), 191-193. Retrieved from https://search.proquest.com/docview/1832679245? accountid=37408

MINISTERIO DE EDUCACIÓN (2009). Diseño Curricular Nacional de la Educación Básica Regular. Lima: Autor. Ministerio de Educación. (2011). Rutas de Aprendizaje ¿Qué y cómo deben aprender nuestros niños? Recuperado el 22 de junio del 2012, de: http://www2.minedo.gob,pe/umc/ece2011/Rutas de aprendizaje/COMO_DEBEN_ APRENDER_NUESTROS_NIÑOS/MATEMATICA17 de mayo1pdf.

Muñiz-Rodríguez L., Alonso P., y Rodríguez-Muñiz, L. (2014). El uso de los juegos como recurso didáctico para la enseñanza y el aprendizaje de las Matemáticas: estudio de una experiencia innovadora. Revista Iberoamerica de Educación Matemática.

Piaget J., y Teóricos A. (1976). Desarrollo cognitivo. España: Fomtaine.

Rodríguez F. (2006). Matematicas estrategias de ensenanza y aprendizaje. Editorial Pax México.

Sallán, J. (1990). Efectos de la utilización de juegos educativos en la enseñanza de las matemáticas. Educar, (17), 105-118.

Sarlé P. (2006). Enseñar el juego y jugar la enseñanza. Buenos Aires: Paidós.

Zapata J., Colorado H., y Gutiérrez H. (2016). El juego como una estrategia didáctica para desarrollar el pensamiento numérico en las cuatro operaciones básicas. Sophia, 12(1), 117-125. 